was migraine (72\%), followed by tension-type headache (56\%). Dizziness and/or vertigo was a commonly reported coexisting symptom, with $24 \%$ reporting this. For $13 \% \quad(n=29)$ of cases, it was documented that time was taken out of work or studies due to headache symptoms. In 205 patients it was reported whether sleep was affected, with $70 \%(n=144)$ of cases indicating it was. For 195 cases, 80\% $(n=155)$ reported a normal appetite, $16 \%(\mathrm{n}=32)$ a decrease, and $4 \%(\mathrm{n}=8)$ an increase in appetite. In 195 patients, 65\% $(n=126)$ reported reduced energy levels. For 21\% $(n=46)$ there was documentation of anxiety. Regarding mood, in 176 cases where this was recorded, 33\% gave a negative mood description.

Conclusion To the authors' knowledge, this study is the first to report on headache characteristics in patients presenting to a UK general neurology clinic. The diagnostic frequency of different headaches presented in this study are comparable to those described in specialist headache clinics. Our population demonstrate significant psychiatric morbidity associated with headaches, with $33 \%$ reporting negative mood value and in addition higher proportions reporting energy and sleep disturbance. To conclude, this study has shown the common headache diagnoses encountered in a general neurology clinic, and indeed is comparable to headaches managed in primary care. Improved integration between these services is key to ensuring effective care for such patients.

\section{9 'NOBODY PREPARED ME FOR THIS!' PARENTS' EXPERIENCES OF SEEKING HELP AND SUPPORT WITH POST-BRAIN INJURY SYMPTOMS AND CHANGES IN CHILDREN AND ADOLESCENTS WITH ACQUIRED BRAIN INJURY}

Robyn MCCarron*

\subsection{6/jnnp-2019-BNPA.19}

Objectives/aims This research aims to:

- Understand the journeys and processes that parents follow in seeking help and support for post-brain injury symptoms and changes in their children.

- Identify the barriers and challenges that parents face in obtaining help and support for their children and family following paediatric Acquired Brain Injury (ABI).

- Propose ways in which services can be more responsive to the evolving needs of children and adolescents with ABI and their families.

Method Five mothers were interviewed using semi-structured interviews about their experiences of seeking help and support for post-brain injury symptoms and changes in their children. The participants' children were 60\% male, 10-16 years old and were 2-6 years post injury. Four had suffered strokes and one had a Traumatic Brain Injury. The age at injury was 4-14 years old. The interviews were audio-recorded and transcribed into 2698 units of data. The data was analysed using a Glaserian grounded theory constant comparative approach.

Results After a child suffers an ABI parents feel that it is down to them to ensure that their child gets the help and support that they need. Identifying and accessing support requires a high level of research and persistence that parents experience as a 'battle'. Obtaining support for mental-health problems is especially challenging. Common barriers include communication issues, time and funding delays, a lack of consistent professionals, not meeting the criteria for services, and a lack of local or age-appropriate services. The challenges of trying to seek help and support impact upon the whole family in terms of time, finances, socially and emotionally. However, through this process parents do become expert parents/carers. They gain new knowledge and language and they look to share this to support other parents going through similar experiences.

Conclusions Providing parents with accessible psychoeducation around post-brain injury symptoms and changes in the acute period following a paediatric ABI could be a helpful intervention to make parents feel more prepared and supported in the medium to long term. More specialist paediatric ABI services that can provide integrated, holistic and developmental care are needed. Current services may need a more flexible and longer-term approach to be responsive to the needs of young people with ABI.

\section{CLINICAL CHARACTERISTICS OF PATHOLOGICAL CONFIRMED EARLY ONSET DEMENTIA WITH LEWY BODIES}

1,2,3Simon Kang Seng Ting ${ }^{*},{ }^{1}$ Celeste Chen, ${ }^{4}$ Huihua Li $1,2,3$ Shahul Hameed, ${ }^{2}$ Adeline NG, 1,2,3 Eng-King Tan, ${ }^{2,5} \mathrm{Kok}$ Pin Ng, ${ }^{2,3}$ Nagaendran Kandiah. ${ }^{1}$ Department of Neurology, Singapore General Hospital, Singapore, Singapore; ${ }^{2}$ Department of Neurology, National Neuroscience Institute, Singapore, Singapore; ${ }^{3}$ Duke-NUS Medical School, Singapore; ${ }^{4}$ Health Services Research and Biostatistics Unit, Singapore General Hospital

\subsection{6/jnnp-2019-BNPA.20}

Background Early-onset dementia (EOD) is characterized by distinct clinical profiles and prognosis when compared to lateonset dementia (LOD). As the second most common neurodegenerative form of dementia, little is known about the clinical profile of early-onset Dementia with Lewy Bodies (DLB). A current challenge for clinicians when managing patients with DLB is the suboptimal diagnosis rate which will affect treatment efficacy and outcome. To address this knowledge gap, by hypothesizing early-onset DLB will have a distinct profile when compared to Alzheimer's disease (AD), we accessed and reviewed data of patients with pathological confirmed DLB from National Alzheimer's Coordinating Center (NACC) database.

Methods Patients with first visit that fulfill criteria for dementia of AD or DLB were analyzed. Early onset age was defined as less than 65 years old. Variables included in the analyses include baseline demographics, cognitive, behavioral, motor symptoms, neuropsychological battery scores and clinician diagnosis. Comparisons were made between early-onset $\mathrm{AD}$ (EOAD) versus early-onset DLB (EODLB), and early versus late-onset DLB.

Results This study included 363 patients with EOAD, 32 EODLB and 147 late-onset DLB. Patients with EODLB were more likely to present with psychosis, apathy, REM sleep behavioral disorder, and motor symptoms. While EOAD patients were more likely to present with cognitive symptoms as first recognized and predominant presentation and perform worse in memory assessment. Motor as first recognized presentation, slowness, visual hallucination, caregiver reporting of agitation and apathy were the significant predictors to differentiate the two. Late-onset DLB patients were less depressed and more impaired in memory and executive function related scores than EODLB. Significant number of EODLB patients were misdiagnosed as $\operatorname{EOAD}(46.9 \%, \mathrm{p}<0.0001)$. 
Conclusions EODLB is characterized by motor and neuropsychiatric symptoms while neuropsychological tests appear less reliable to differentiate EODLB from EOAD. Given that misdiagnosis of DLB remain significantly high, we propose a more careful and comprehensive clinical approach may improve the diagnosis rate.

Acknowledgement The NACC database is funded by NIA/NIH Grant U01 AG016976. NACC data are contributed by these NIA funded ADCs: P30 AG019610 (PI Eric Reiman, MD), P30 AG013846 (PI Neil Kowall, MD), P50 AG008702 (PI Scott Small, MD), P50 AG025688 (PI Allan Levey, MD, PhD), P50 AG047266 (PI Todd Golde, MD, PhD), P30 AG010133 (PI Andrew Saykin, PsyD), P50 AG005146 (PI Marilyn Albert, PhD), P50 AG005134 (PI Bradley Hyman, MD, PhD), P50 AG016574 (PI Ronald Petersen, MD, PhD), P50 AG005138 (PI Mary Sano, PhD), P30 AG008051 (PI Steven Ferris, PhD), P30 AG013854 (PI M. Marsel Mesulam, MD), P30 AG008017 (PI Jeffrey Kaye, MD), P30 AG010161 (PI David Bennett, MD), P50 AG047366 (PI Victor Henderson, MD, MS), P30 AG010129 (PI Charles DeCarli, MD), P50 AG016573 (PI Frank LaFerla, PhD), P50 AG016570 (PI Marie-Francoise Chesselet, MD, PhD), P50 AG005131 (PI Douglas Galasko, MD), P50 AG023501 (PI Bruce Miller, MD), P30 AG035982 (PI Russell Swerdlow, MD) , P30 AG028383 (PI Linda Van Eldik, PhD), P30 AG010124 (PI John Trojanowski, MD, PhD), P50 AG005133 (PI Oscar Lopez, MD), P50 AG005142 (PI Helena Chui, MD), P30 AG012300 (PI Roger Rosenberg, MD), P50 AG005136 (PI Thomas Montine, MD, PhD), P50 AG033514 (PI Sanjay Asthana, MD, FRCP), P50 AG005681 (PI John Morris, MD), and P50 AG047270 (PI Stephen Strittmatter, MD, PhD).

This study is supported by Singhealth Foundation Grant (NRS 15/001), NNI Centre Grant (NCG CS02) and National Medical Research Council, Singapore (NMRC/IRG/015).

\section{UNDERSTANDING FOREIGN ACCENT SYNDROME}

${ }^{1}$ Laura McWhirter ${ }^{*},{ }^{2}$ Nick Miller, ${ }^{3}$ Catriona Campbell, ${ }^{1}$ Ingrid Hoeritzauer, ${ }^{4}$ Andrew Lawton, ${ }^{1}$ Alan Carson, ${ }^{1} J$ Jon Stone. ${ }^{1}$ Centre for Clinical Brain Sciences, University of Edinburgh; ${ }^{2}$ Speech and Language Sciences, University of Newcastle upon Tyne; ${ }^{3}$ Department of Clinical Psychological Science, Maastricht University; ${ }^{4}$ Department of Psychological Medicine, Royal Infirmary of Edinburgh

\subsection{6/jnnp-2019-BNPA.21}

Objectives/Aims Foreign accent syndrome (FAS) is a disorder of speech in which listeners perceive the affected individual as speaking with a foreign or different regional accent that is not their habitual accent. FAS is widely understood as an unusual consequence of stroke or other lesions within speech-motor networks. However, case reports of FAS occurring in the absence of structural damage and difficulty identifying neural correlates has led to increasing recognition that that FAS sometimes represents a functional neurological disorder. We aimed to characterise symptoms, comorbidities, and features of recorded speech in individuals with selfreported FAS.

Methods Participants self-reporting FAS recruited from informal unmoderated online support forums and recruited via professional networks completed an online survey. Recorded samples of spontaneous speech and reading of a standardised text were analysed in a subgroup of 13 cases.

Results Forty-nine respondents (24 UK, 23 North America, 2 Australia) reported FAS of mean duration three years (range two months - 18 years). Common triggers were: migraine/ severe headache (15), stroke (12), surgery or injury to mouth or face (six), and seizure (five, including three non-epileptic). High levels of comorbidity included migraine (33), irritable bowel syndrome (17), functional neurological disorder (12), and chronic pain (12). Five reported structural lesions on imaging. Author consensus on aetiology divided into, 'probably functional' $(n=35,71 \%)$, 'possibly structural' $(n=4,8 \%)$, and 'probably structural'( $\mathrm{n}=10,20 \%)$, but positive features of functional FAS were present in all groups. Blinded analysis of speech recordings supplied by 13 respondents correctly categorised $11(85 \%)$ based on probable aetiology (functional vs structural) in agreement with case history assignment. Analysis of speech recordings identified a range of features demonstrating internal inconsistency with potential utility in the diagnosis of functional FAS.

Conclusions This study, of the largest FAS case series to date, details the experience and characteristics of individuals with self-reported FAS, and describes an approach to auditory-perceptual analysis of speech with potential diagnostic utility. Although conclusions are limited by the recruitment methods, high levels of functional comorbidity, symptom variability and additional linguistic and behavioural features suggest that chronic FAS may in many cases represent a functional neurological disorder, even when a structural lesion is present.

\section{MISSED DIAGNOSIS OF ADHD IN CHILDREN REFERRED TO A TIC DISORDER CLINIC}

Idura N Hisham*, Jeremy S Stern, Helen Simmons. St George's Hospital and St George's University of London

\subsection{6/jnnp-2019-BNPA.22}

Aim To examine whether Attention Deficit Hyperactive Disorder (ADHD), a common comorbid disorder in Tourette's Syndrome (TS) patients, is often missed by physicians referring to a Tic Disorder Clinic.

Method Referral letters and first clinic attendance reports for 119 new patients aged between 4-17 that attended a national tic disorder clinic between 2015-2017 were analysed to see how many new diagnoses of ADHD were made at first consultation that were not included in the referral letters. Other variables that were noted for each patient included age, sex, if referrer had a suspicion of ADHD (rather than established or firm diagnosis), medication for $\mathrm{ADHD}$ and the main treatment target decided at the tic disorder clinic.

Results Out of 119 patients 13 (11\%) already had a diagnosis of ADHD, which is in line with the prevalence of comorbid $\mathrm{ADHD}$ in the general population but not with the known increased prevalence in patients with TS (up to $80 \%$ in some studies). The assessment at the Tic Disorder Clinic found 46 cases of ADHD (38\%). Referrals were from pediatricians (51\%), general practitioners (35\%) and from mental health services $(10 \%)$.

Conclusions As the prevalence of comorbid ADHD is high in Tourette's patients and this can sometimes be obscured by the presentation of the tic disorder, referrers should have a low threshold for suspecting and managing ADHD in cases where specialist input for tics is awaited. It is likely that CAMHS referrals were under-represented in the sample and it may be expected that prior ADHD diagnoses would be more likely from that source. 